Pacific Journal of Mathematics

CONTINUITY OF TRANSFORMATIONS WHICH LEAVE
INVARIANT CERTAIN TRANSLATION INVARIANT SUBSPACES 


\title{
CONTINUITY OF TRANSFORMATIONS WHICH LEAVE INVARIANT CERTAIN TRANSLATION INVARIANT SUBSPACES
}

\author{
B. E. JoHNSON
}

\begin{abstract}
It is shown that a linear operator $T: L^{2}(X) \rightarrow L^{2}(X)(X$ a locally compact group), with the property that $T E \subset E$ for each norm closed right translation invariant subspace $E$ of $L^{2}(X)$, is necessarily continuous. In $\S 5$ the author shows that this is also true for $L^{1}(X)$ when $X$ contains an element $a$ which does not lie in any compact subgroup. An example is constructed to show that, in $l^{\infty}(-\infty,+\infty), T$ can be discontinuous and still leave invariant each $\sigma\left(l^{\infty}, l^{1}\right)$ closed translation invariant subspace of $l^{\infty}$. If however $T: l^{\infty}(-\infty,+\infty) \rightarrow$ $l^{\infty}(-\infty,+\infty)$ leaves invariant all norm closed translation invariant subspaces, then $T$ must be continuous.
\end{abstract}

We shall use the notation of [3] without further explanation. Sections 3, 4 and 5 overlap with some of the results in 1.4 of Edwards' paper [3] where he shows inter alia that $T$ is automatically continuous in the $L^{2}$ case if $X$ is compact and in the $L^{1}$ case if $X$ is finitely representable. The work in $\S 6$ answers the conjecture in 1.7 of [3].

2. Three basic lemmas. For the first lemma let $\mathfrak{X}$ be a normal topological space and $\mathscr{A}$ a set of complex valued functions on $\mathfrak{X}$, closed with respect to pointwise multiplication, containing the constant function 1 and such that if $f \in \mathscr{A}$ then so is $1-f$. We assume that $\mathscr{A}$ is normal in the sense that if $F_{0}$ and $F_{1}$ are closed disjoint subsets of $\mathfrak{X}$ then there is a function $f \in \mathscr{A}$ with $f\left(F_{0}\right)=\{0\}, f\left(F_{1}\right)=$ $\{1\}$. We suppose that there is a mapping $f \rightarrow S_{f}$ of $\mathscr{A}$ into $\mathscr{B}(E)$, where $E$ is some Banach space, such that $S_{f g}=S_{f} S_{g}, S_{1}=I, S_{1-f}=$ $I-S_{f}$. We denote the null space of $S_{f}$ by $N_{f}$ and suppose that $T$ is a linear map of $E$ into $E$ which leaves invariant all the subspaces $N_{f}(f \in \mathscr{A})$. For each $f \in \mathscr{A}$ the composition of $T$ with the quotient map $E \rightarrow E / N_{f}$ gives a map $T_{f}: E \rightarrow E / N_{f}$.

Definition 2.1. $\lambda \in \mathfrak{X}$ is called a discontinuity value of $T$ if $T_{f}$ is discontinuous whenever $f \in \mathscr{A}$ and there exists a neighbourhood $N$ of $\lambda$ such that $f\left(\lambda^{\prime}\right) \neq 0$ for all $\lambda^{\prime} \in N$.

Lemma 2.2. Thas only a finite number of discontinuity values.

Proof. Suppose $T$ has a infinity of discontinuity values. Then 
we can find a sequence $\lambda_{i}$ of such values and a closed neighbourhood $V_{i}$ of each such that, for each $i, V_{i}$ and $\left[\bigcup_{j \neq i} V_{j}\right]^{-}$are disjoint. For each $i$ let $f_{i}$ be a function in $\mathscr{A}$ with $f_{i}\left(V_{j}\right)=\delta_{i, j}$ and let $g_{i} \in \mathscr{A}$ such that $g_{i}(\lambda)=0$ for $\lambda \notin V_{i}$ and $g_{i}(\lambda) \neq 0$ for all $\lambda$ in some neighbourhood of $\lambda_{i}$. Then take $\xi_{i} \in E$ such that $\left\|\xi_{i}\right\| \cdot\left\|S_{f_{i}}\right\|<2^{-i}$ and $\left\|T_{g_{i}} \xi_{i}\right\|>2^{i}$. Put $\eta=\sum S_{f_{i}} \xi_{i}$. Then $S_{g_{j}} S_{f_{i}}=0$ for $j \neq i$ so that $\sum_{i \neq j} S_{f_{i}} \xi_{i} \in N_{g_{j}}$, and $S_{g_{j}} S_{1-f_{j}}=0$ so that $\xi_{j}-S_{f_{j}} \xi_{j} \in N_{g_{j}}$. Thus

$$
\|T \eta\| \geqq\left\|T_{g_{j}} \eta\right\|=\left\|T_{g_{j}} S_{f_{j}} \xi_{j}\right\|=\left\|T_{g_{j}} \xi_{j}\right\|>2^{j}
$$

for all $j$, which is impossible.

Lemma 2.3. Let $A$ be an index set and let, for each $\alpha \in A, E_{\alpha}$ be a Banach space and $P_{\alpha}$ a continuous linear map from $E$ into $E_{\alpha}$ with null space $N_{\alpha}$. Let $N=\cap N_{\alpha}$ and let $P$ be the quotient map $E \rightarrow E / N$. Let $T$ be a linear map $E \rightarrow E$ such that $P_{\alpha} T$ is continuous for each $\alpha \in A$. Then PT is continuous.

Proof. Suppose $x_{n} \rightarrow 0$ in $E$ and $P T x_{n} \rightarrow y$ in $E / N$. Since $N \subset N_{\alpha}$ the $P_{\alpha}$ give rise to maps $Q_{\alpha}: E / N \rightarrow E_{\alpha}$ and $Q_{\alpha} P T=P_{\alpha} T$. Hence $Q_{\alpha} y=\lim _{n} Q_{\alpha} P T x_{n}=\lim _{n} P_{\alpha} T x_{n}=0$. Thus $Q_{\alpha} y=0$ for all $\alpha \in A$ which implies that $y=0$. Hence, by the closed graph theorem, $P T$ is continuous.

LEMMA 2.4. Let $H$ be a Hilbert space, $\mathfrak{X}$ a locally compact Hausdorff space, $P($.$) a spectral measure on \mathfrak{X}$ (see [5], p. 246), $\mathscr{G}$ a collection of open sets from $\mathfrak{X}, N=\bigcap_{\epsilon \in \mathscr{G}} N(P(G))$ and $P_{0}$ the orthogonal projection with null space $N$. Then $P_{0}=P(\cup \mathscr{G})$.

Proof. With the usual ordering of projections $P(\cup \mathscr{G}) \geqq P(G)$ for each $G$ in $\mathscr{G}$ and so $P(\cup \mathscr{G}) \geqq P_{0}$. Now let $\xi_{0} \in N$ and consider the measure $\mu(Y)=\left(P(Y) \xi_{0}, \xi_{0}\right)$ defined on the borel sets in $\mathfrak{X}$. Since $\mu(G)=0$ for all $G$ in $\mathscr{G}, \cup \mathscr{G}$ is locally of $\mu$ measure zero and hence since it is open, $\mu(\cup \mathscr{G})=0$ (see [1], p. 183). However this implies $P(\cup \mathscr{G}) \xi_{0}=0$ and so $P_{0}=P(\cup \mathscr{G})$.

\section{3. $L^{2}(X)$, the simple case.}

THEOREM 3.1. Let $X$ contain an element a which is not contained in any compact subgroup. Let $T$ be a linear map $L^{2}(X) \rightarrow L^{2}(X)$ which leaves invariant each closed right translation invariant subspace of $L^{2}(X)$. Then $T$ is continuous.

Proof. Apply Lemma 2.2 with $\mathfrak{X}$ as the circle $|z|=1$ in $C$ and $\mathscr{A}$ the set of characteristic functions of measurable sets in $\mathfrak{X}$. The 
operator $\tau_{a}$ is a unitary operator in $L^{2}(X)$ and its spectral resolution $P($.$) gives the required map from \mathscr{A}$ into $\mathscr{B}\left(L^{2}(X)\right)$. Since the operators corresponding to elements of $\mathscr{A}$ are weak operator limits of polynomials in $\tau_{a}$ and $\tau_{a}^{*}$, they commute with all right translations. In particular the ranges and null spaces of the values of $P($.$) are$ closed right translation invariant subspaces.

In the following it will be convenient not to distinguish too carefully between the quotient of a Hilbert space by a closed subspace and the orthogonal complement of the subspace.

Application of 2.2 shows that $T$ has only finitely many discontinuity values $\lambda_{1}, \cdots, \lambda_{k}$. For each $\lambda \in \mathfrak{X}$ which is not a discontinuity value we thus have a set $Y \subset \mathfrak{X}$ containing $\lambda$ as an interior point such that $P(Y) T$ is continuous; clearly $Y$ can be taken to be open. Application of 2.3 taking $A$ as the class of open sets $G$ in $\mathfrak{X}$ with $P(G) T$ continuous and $P_{G}=P(G)$ shows that $P_{0} T$ is continuous where $P_{0}$ is the orthogonal projection along the intersection of the null spaces of the $P(G) ; G \in A$. Lemma 2.4 then shows that $P_{0}=I-P\left(\left\{\lambda_{1}\right\}\right)-\cdots-P\left(\left\{\lambda_{l k}\right\}\right)$.

If now $\xi \neq 0$ is in the range of $P\left(\left\{\lambda_{1}\right\}\right)$ then $\tau_{\alpha} \xi=\lambda_{1} \xi$. We can find a compact set $K$ such that $\int_{K}|\xi(x)|^{2} d x>1 / 2$. $\|\xi\|^{2}$ and an integer $n$ such that $a^{n} \notin K K^{-1}$. Thus

$$
\begin{aligned}
\text { 1/2. }\|\xi\|^{2} & <\int_{K}|\xi(x)|^{2} d x \\
& =\int_{K}\left|\lambda_{1}^{n} \xi(x)\right|^{2} d x \\
& =\int_{K}\left|\xi\left(a^{-n} x\right)\right|^{2} d x \\
& =\int_{a^{-n_{K}}}|\xi(x)|^{2} d x .
\end{aligned}
$$

Since $a^{-n} K \cap K$ is void this gives

$$
\begin{aligned}
\|\xi\|^{2} & \geqq \int_{K}|\xi(x)|^{2} d x+\int_{a-x_{K}}|\xi(x)|^{2} d x \\
& >1 / 2 .\|\xi\|^{2}+1 / 2 .\|\xi\|^{2} .
\end{aligned}
$$

This contradiction shows that the range of $P\left(\left\{\lambda_{1}\right\}\right)$ is the zero subspace and $P\left(\left\{\lambda_{1}\right\}\right)=0$. Similarly $P\left(\left\{\lambda_{2}\right\}\right)=\cdots P\left(\left\{\lambda_{k}\right\}\right)=0, P_{0}=I$ and $T$ is continuous.

REMARK 3.2. A stronger result would be obtained if we could consider operators reduced by subspaces closed in some topology weaker than the norm topology. Since $L^{2}$ is reflexive there is no advantage in considering the weak topology. The result is untrue if we take the topology $\sigma\left(L^{2}, L_{0}\right)$ where $L_{0}$ is the subspace of $L^{2}$ consisting of 
functions with compact support since, in the case $X=Z$, the additive group of the integers, there are no $\sigma\left(L^{2}, L_{0}\right)$ - closed translation invariant proper subspaces of $L^{2}$. In this case if $\xi \in L_{0}, \xi \neq 0$ then for $\eta$ and all its translates to be in $\xi^{\perp}$ we must have $\xi^{*} \eta=0$, or $\hat{\xi} \hat{\eta}=0$ where $\hat{\xi}, \hat{\eta}$ are the functions in $L^{2}$ of the unit circle whose Fourier coefficients are $\xi, \eta$. Since $\hat{\xi}$ is a polynomial this implies that $\hat{\eta}=0$ almost everywhere and so $\eta=0$.

REMARK 3.3. If $X$ is not unimodular or has a nonunimodular subgroup then it satisfies the condition of the theorem.

4. $L^{2}(X)$, the general case. The methods of [3] apply when the group $X$ is finitely representable. To show that there is indeed ground not covered by either Edwards' method or Theorem 3.1 we take the group in the following example with the discrete topology.

EXAMPLE 4.1. The alternating group $A$ on to symbols (see [4], p. 40) is not finitely representable yet every element lies in a finite subgroup.

Proof. We consider the permutations as acting on 1,2,3, .. and denote by $A_{n}$ the subgroup leaving $n+1, n+2, \cdots$ invariant. $A_{n}$ is isomorphic with the alternating group on $n$ symbols in an obvious way. Suppose $\varphi: A \rightarrow G L(m)$ is an $m$ dimensional representation of $A$. Then $\varphi$ induces $m$ dimensional representations of $A_{n}$ for each $n$ which must be trivial for $n>m+1$ (see [2], p. 466). Since $A=\cup A_{n}, \varphi$ is trivial on $A$. Thus there is no nontrivial finite dimensional representation of $A$.

THEOREM 4.2. Let $X$ be locally compact but not compact. Let $T$ be a linear map $L^{2}(X) \rightarrow L^{2}(X)$ which leaves invariant each closed right translation invariant subspace of $L^{2}(X)$. Then $T$ is continuous.

Proof. We use here ideas occuring in the theory of $W^{*}$-algebras (see e.g. [5], §41).

Let $\&$ be the set of elements of $\mathscr{B}\left(L^{2}\right)$ commuting with all the right translation operators. The range and null space of any projection in $\mathbb{R}$ is closed with respect to right translation and hence each such projection commutes with $T$. We consider $Z$, the centre of $\mathbb{R}$, a commutative $W^{*}$-algebra. Let $\mathscr{C l}$ be its maximal ideal space so that $\mathscr{C l}$ is an extremally disconnected compact topological space (see [6], p. 294). To show that $T$ has only finitely many discontinuity values $\lambda_{1}, \cdots, \lambda_{k}$, we then apply Lemma 2.2 with $\mathscr{l l}$ as $\mathfrak{X}, \mathscr{A}$ as the set of characteristic functions of open closed sets in $\mathfrak{X}$ and for $f \in \mathscr{A}$, 
$S_{f}$ as the projection in $Z$ whose Gelfand transform is $f$.

Applying 2.3 with $A$ as the set of open closed sets $G$ in $/ /$ for which $P(G) T$ is continuous, where $P(G)$ is the element of $Z$ whose Gelfand transform is the characteristic function of $G$, we obtain a projection $P \geqq P(G)$ for all $G$ in $A$, such that $P T$ is continuous. From the way in which it is constructed, $P \in Z$, and so $P=P\left(G_{0}\right)$ where $G_{0}$ is the maximum element in $A$ which must be $\mathscr{C l} \backslash\left\{\lambda_{1}, \cdots, \lambda_{k}\right\}$. This shows that $\left\{\lambda_{1}, \cdots, \lambda_{k}\right\}$ is open closed and, since $\mathscr{C}$ is Hausdorff, that if $T$ is discontinuous then $\mathscr{C l}$ has isolated points.

Let now $\lambda_{0} \in\left\{\lambda_{1}, \cdots, \lambda_{k}\right\}$ and let $H_{0}$ be the range of the corre-. sponding projection $P_{0}=P\left(\left\{\lambda_{0}\right\}\right)$.

The algebras $\mathfrak{L}_{0}, \mathfrak{L}_{0}^{\prime}$ formed by restricting the operators in $\mathfrak{R}, \mathbb{R}^{\prime}$ to $H_{0}$ form a factored pair (see [5], p. 450). Now let $C$ be a maximal commutative subalgebra of $\Omega_{0}$. Application of the argument in the previous paragraphs to $C$ in place of $Z$ shows that the maximal ideal space of $C$ contains at least one isolated point $\mu$. Let $M_{0}$ be the range of the corresponding projection $Q$. Since $Q \in \mathbb{R}_{0}, M_{0}$ is a subspace adjoined to $\mathbb{S}_{0}$ and is moreover a minimal subspace ([5], p. 451). It follows that the factor $\mathbb{R}_{0}$ is of type $I$. The structure of such factors is as follows ([5], p. 480). $H_{0}$ is a direct sum of orthogonal subspaces $M_{\alpha} ; \alpha \in A$, each isomorphic with $M_{0}$ (and which we shall consider as copies of $M_{0}$ ). Relative to this decomposition of $H_{0}$ the matrix of an element of $\mathscr{R}_{0}^{\prime}$ is $\left[\delta_{\alpha, \beta} A_{0}\right]$ where $A_{0} \in \mathscr{B}\left(M_{0}\right)$. This is an isomorphism between $\mathfrak{R}_{0}^{\prime}$ and $\mathscr{B}\left(M_{0}\right)$. The matrix of an element of $\mathfrak{L}_{0}$ is $\left[\alpha_{\alpha, \beta} I_{0}\right]$ where the $a_{\alpha, \beta}$ are complex numbers and $I_{0}$ is the identity operator in $M_{0}$. This is an isomorphism between $\mathbb{Z}_{0}$ and those matrices of the form $\left[\alpha_{\alpha, \beta} I_{0}\right]$ giving bounded transformations.

Applying the results in $\S 2$ a third time we see that there is only a finite number of values $\alpha_{1}, \cdots, \alpha_{n}$ of $\alpha$ for which the restriction of $T$ to $M_{\alpha}$ is not continuous. Let, if possible, $\alpha_{0}$ be a value of $\alpha$ different from $\alpha_{1}, \cdots, \alpha_{n}$. Let $P$ be the operator in $\mathfrak{\Omega}_{0}$ whose matrix $\left[a_{\alpha, \beta} I_{0}\right]$ is given by $a_{\alpha_{1}, \alpha_{0}}=a_{\alpha_{1}, \alpha_{1}}=1, a_{\alpha, \beta}=0$ otherwise. $P$ is a nonorthogonal projection and $P T_{0}=T_{0} P$ (where $T_{0}$ is the restriction of $T$ to $H_{0}$ ) so that $P T_{0} M_{\alpha_{1}}=T_{0} P M_{\alpha_{1}}=T_{0} M_{\alpha_{0}}$ which is impossible since $P$ maps $M_{\alpha_{1}}$ isometrically onto $M_{\alpha_{0}}$ whilst $T_{0}$ is bounded on $M_{\alpha_{0}}$ and unbounded on $M_{\alpha_{1}}$. Thus $A=\left\{\alpha_{1}, \cdots, \alpha_{n}\right\}$.

Now let $\xi_{0} \in M_{0}$ with $\left\|\xi_{0}\right\|=1$ and let $\xi_{1}, \cdots, \xi_{n}$ be its copies in $M_{\alpha_{1}}, \cdots, M_{a_{n}}$. The subspace of $L^{2}(X)$ generated by $\xi_{1}, \cdots, \xi_{n}$ is closed with respect to all operators in $\&$. Let $K$ be a compact set in $X$ such that

$$
\int_{K}\left|\xi_{i}(t)\right|^{2} d t>1-(2 n)^{-2}
$$

for $i=1, \cdots, n$ and let $a \in X \backslash K K^{-1}$ (such an element exists since $K K^{-1}$ is compact and $X$ is not). Then $\tau_{a} \xi_{1}=a_{1} \xi_{1}+\cdots+a_{n} \xi_{n}$ where 
$\left|a_{1}\right|^{2}+\cdots+\left|a_{n}\right|^{2}=1$. Let $c$ be the characteristic function of $a K$. Then

$$
\begin{aligned}
\left\|\left(\tau_{a} \xi_{1}\right) c\right\|^{2} & =\int_{K}\left|\xi_{1}(t)\right|^{2} d t \\
& >3 / 4
\end{aligned}
$$

whereas

$$
\begin{aligned}
\left\|\xi_{i} c\right\|^{2} & \leqq \int_{X \backslash K}\left|\xi_{i}(t)\right|^{2} d t \\
& \leqq(2 n)^{-2}
\end{aligned}
$$

so that

$$
\begin{aligned}
\left\|\left(\tau_{a} \xi_{1}\right) c\right\| & =\left\|a_{1} \xi_{1} \cdot c+\cdots+a_{n} \xi_{n} \cdot c\right\| \\
& \leqq\left|a_{1}\right| \cdot\left\|\xi_{1} \cdot c\right\|+\cdots+\left|a_{n}\right| \cdot\left\|\xi_{n} \cdot c\right\| \\
& \leqq 1 / 2
\end{aligned}
$$

which is a contradiction, proving the theorem.

\section{5. $L^{1}(X)$, the simple case.}

THEOREM 5.1. Let $X$ contain an element a which is not contained in any compact subgroup. Let $T$ be a linear operator from $L^{1}(X)$ into $L^{1}(X)$ such that $\mu^{*} T f=0$ whenever $\mu \in M_{b d}(X), f \in L^{1}(X)$ with $\mu^{*} f=0$. Then $T$ is continuous.

Proof. As in 3.1 take $\mathfrak{X}$ as the circle $|z|=1$ and $\mathscr{A}$ as the set of functions $f$ on $\mathfrak{X}$ with absolutely convergent Fourier series $\sum a_{n} e^{i n \theta}$. We take for $S_{f}$ the operator $g \rightarrow \mu_{f}^{*} g$ where, for $f \in \mathscr{A}$ $\mu_{f}=\sum a_{i}\left(\delta_{a}\right)^{i}$. By Lemma 2.2, $T$ has only finitely many discontinuity values $\lambda_{1}, \cdots, \lambda_{n}$. Let $\mathscr{A}_{0}$ be the subset of $\mathscr{A}$ consisting of those elements $f$ for which $T_{f}$ is continuous. For each $\lambda \in \mathfrak{X}$ different from $\lambda_{1}, \cdots, \lambda_{n}$ there is $f \in \mathscr{A}_{0}$ such that $T_{f}$ is continuous and $f(\lambda) \neq 0$. Put $N=\cap N_{f}\left(f \in \mathscr{A}_{0}\right)$. If $\left\{\beta_{j}\right\} \in l^{1}(-\infty,+\infty)$ with $\hat{\beta} f=0$ for all $f$ in $\mathscr{A}_{0}$ where $\widehat{\beta}=\sum \beta_{j} z^{j}$ then $\beta_{j}=0$ for all $j$ and using the fact that $\mathfrak{X} \backslash\left\{\lambda_{1}, \cdots, \lambda_{n}\right\}$ is $\sigma$-compact, we can find a countable subset $\mathscr{A}_{*}$ of $\mathscr{A}_{0}$ with this property. Now if $g \in N$, then for all $\sum a_{n} z^{n} \in \mathscr{A}_{0}$, $\left(\mu^{*} g\right)(t)=\sum a_{n} g\left(a^{-n} t\right)=0$ for almost all $t$ in $X$. Putting $\beta_{t, k}=g\left(\alpha^{k} t\right)$, $k=\cdots,-1,0,1, \cdots$ we have, for each $f \in \mathscr{A}_{0}, \widehat{\beta}_{t} f=0$ for almost all $t$ in $\mathfrak{X}$. Since $\mathscr{A}_{*} \subset \mathscr{A}_{0}$ and $\mathscr{A}_{*}$ is countable, we have that for almost all $t$ in $\mathfrak{X}, \widehat{\beta}_{t} f=0$ for all $f \in \mathscr{A}_{*}$. Thus $\beta_{t}=0$ for almost all $t$ in $\mathfrak{X}$ and, in particular, $\beta_{t, 0}=g(t)=0$ a.e. in $\mathfrak{X}$ so that $N=\{0\}$. Using 2.3 with $A=\mathscr{A}_{0}$ and $P_{f}$ as the quotient map $L^{1}(X) \rightarrow L^{1}(X) / N_{f}$, the proof is complete. 
REMARK 5.2. The author has not been able to extend this result to the general case.

REMARK 5.3. The condition $\mu^{*} T f=0$ whenever $\mu^{*} f=0$ is of course equivalent to saying that the spaces $N_{\mu}=\left\{f ; f \in L^{1}(X), \mu^{*} f=0\right\}$ are closed with respect to $T$. These spaces are right translation closed and also closed in a variety of topologies including $\sigma\left(L^{1}, C_{0}\right)$ or any other topology $\sigma\left(L^{1}, F\right)$ where $F$ is a subspace of $L^{\infty}$ total over $L^{1}$ and closed with respect to convolution with bounded measures.

6. The space $l^{\infty}$. We now show that the continuity hypothesis in 1.6 of [3] is necessary in the case $X=Z$ (the additive group of integers). We use the results in Ch. VII, $\S 1$ of [7].

ExAmple 6.1. The elements $a=\left\{a_{n}\right\}$ of $l^{\infty}(-\infty,+\infty)$ can be considered as Fourier coefficients of certain distributions $\hat{a}$ on the circle group $|z|=1$ and since $\left\{a_{n}\left(1+n^{2}\right)^{-1}\right\} \in l^{1}(-\infty,+\infty)$ these distributions are, at worst, of order two. If $b=\left\{b_{n}\right\}$ is a sequence of rapid decrease it is the sequence of Fourier coefficients of an infinitely differentiable function $\hat{b}$ on the circle and we have $a^{*} b \in l^{\infty},\left(a^{*} b\right)^{\wedge}=\hat{a} \cdot \hat{b}$. It follows from this and the ideas of spectral synthesis, that the constant sequence 1 lies in the $\sigma\left(l^{\infty}, l^{1}\right)$-closed linear subspace of $l^{\infty}$ spanned by the translates of $a$ if and only if 0 lies in the support of $\hat{a}$. For each positive integer $n$ let $E_{n}=\left\{a ; a \in l^{\infty}, d(0\right.$, supp $\left.\hat{a}) \geqq 1 / n\right\}$. The $E_{n}$ are norm closed subspaces of $l^{\infty}, E_{n} \nsubseteq E_{n+1}$ so that $E_{\infty}=\cup E_{n}$ is of first category with the induced $l^{\infty}$ topology and so is not closed in $l^{\infty}$. We can thus find a discontinuous functional $f$ on $l^{\infty}$ which is 0 on $E_{\infty}$. If we then put $T a=f(a) 1$ we then have a discontinuous linear transformation $T$ on $l^{\infty}$ such that $T a$ is in the $\sigma\left(l^{\infty}, l^{1}\right)$-closure of linear combinations of translates of $a$.

6.2. The conjecture in 1.7 of [3] can only break down in a way similar to that in 6.1. This can be seen by application of the results in $\S 2$ taking $\mathfrak{X}$ as $|z|=1$ and $\mathscr{A}$ as the set of infinitely differentiable functions on $\mathfrak{X}$. We see that if $T$ is a linear transformation $l^{\infty} \rightarrow l^{\infty}$ such that $T a$ is a $\sigma\left(l^{\infty}, l^{1}\right)$-limit of linear combinations of translations of $a$ then there is a subspace $N$ such that $P T$ is continuous where $P$ is the quotient map $l^{\infty} \rightarrow l^{\infty} / N$. The space $N$ is the intersection of null spaces corresponding to a subset $\mathscr{A}_{0}$ of $\mathscr{A}$, the elements of $\mathscr{A}_{0}$ having only a finite number of common zeros $\lambda_{1}, \cdots, \lambda_{n}$. Thus $N$ consists only of elements $a$ with Supp $\hat{a} \subset\left\{\lambda_{1}, \cdots, \lambda_{l}\right\}$ and since $a$ is of order $\leqq 2$ we have

$$
a_{n}=\alpha_{l} \lambda_{l}^{n}+\cdots+\alpha_{l} \lambda_{l}^{n}+n\left(\beta_{l} \lambda_{l}^{n}+\cdots\right) .
$$


The coefficient of $n$ is almost periodic so that for $a$ to be bounded the $\beta_{i}$ are all zero. Hence $N$ is a space of linear combinations of the $\lambda_{j}^{n}, j=1, \cdots, l$.

6.3. If $T$ is assumed to leave invariant all norm closed translation invariant subspaces of $l^{\infty}$ then it is continuous. To show this we apply the argument in 6.2 and then put

$$
\begin{array}{lll}
l^{+}=\left\{a ; a \in l^{\infty}, a_{n}=0\right. & \text { for } & n<0\} \\
l^{-}=\left\{a ; a \in l^{\infty}, a_{n}=0\right. & \text { for } & n \geqq 0\} .
\end{array}
$$

Let $K$ be the norm of $P T$. If $a \in l^{\infty}$ then $a=a^{+}+a^{-}$with $a^{+} \in l^{+}$, $a^{-} \in l^{-}$. Then $\left\|P T a^{+}\right\| \leqq K\left\|a^{+}\right\| \leqq K\|a\|$ and so there is $b \in N$ such that $\left\|T a^{+}-b\right\| \leqq 2 K\|a\|$. However since $T a^{+}$is a uniform limit of linear combinations of translates of $a^{+}$, and hence $\left(T a^{+}\right)_{n} \rightarrow 0$ as $n \rightarrow-\infty$, and $b$ is almost periodic we have $\|b\| \leqq\left\|T a^{+}-b\right\|$. From this we see that $\left\|T a^{+}\right\| \leqq\left\|T a^{+}-b\right\|+\|b\| \leqq 4 K\|a\|$, and applying the same argument to $\left\|T a^{-}\right\|$we get a similar conclusion, the two together giving $\|T a\| \leqq 8 K\|a\|$.

\section{REFERENCES}

1. N. Bourbaki, Éléments de mathématique, intégration, Ch. I-IV, Act. sci. ind. 1175, Hermann, Paris, 1952.

2. W. Burnside, Theory of groups of finite order, 2nd ed., Cambridge, 1911.

3. R. E. Edwards, Endomorphisms of function-spaces which leave stable all translationinvariant manifolds, Pacific J. Math. 14 (1964), 31-48.

4. A. G. Kurosh, Theory of groups, Vol. I, Chelsea, New York, 1955.

5. N. A. Naimark, Normed rings, Noordhoff, Groningen, 1959.

6. C. E. Rickart, General theory of banach algebras, Van Nostrand, New York, 1960.

7. L. Schwartz, Théorie des distributions, Tome II, Act. sci. ind. 1122, Hermann, Paris, 1951.

Received October 8, 1965.

The UnIVersity

NewCastle Upon Tyne

ENGLAND 


\section{PACIFIC JOURNAL OF MATHEMATICS}

\section{EDITORS}

\author{
H. SAMELSON \\ Stanford University \\ Stanford, California \\ J. P. JANS \\ University of Washington \\ Seattle, Washington 98105
}

\section{J. DugundJI}

University of Southern California Los Angeles, California 90007

RICHARD ARENS

University of California

Los Angeles, California 90024

\section{ASSOCIATE EDITORS}
E. F. BECKENBACH
B. H. NeumanN
F. WOLF
K. YOSIDA

\section{SUPPORTING INSTITUTIONS}

\author{
UNIVERSITY OF BRITISH COLUMBIA \\ CALIFORNIA INSTITUTE OF TECHNOLOGY \\ UNIVERSITY OF CALIFORNIA \\ MONTANA STATE UNIVERSITY \\ UNIVERSITY OF NEVADA \\ NEW MEXICO STATE UNIVERSITY \\ OREGON STATE UNIVERSITY \\ UNIVERSITY OF OREGON \\ OSAKA UNIVERSITY \\ UNIVERSITY OF SOUTHERN CALIFORNIA
}

\author{
STANFORD UNIVERSITY \\ UNIVERSITY OF TOKYO \\ UNIVERSITY OF UTAH \\ WASHINGTON STATE UNIVERSITY \\ UNIVERSITY OF WASHINGTON \\ AMERICAN MATHEMATICAL SOCIETY \\ CHEVRON RESEARCH CORPORATION \\ TRW SYSTEMS \\ NAVAL ORDNANCE TEST STATION
}

Mathematical papers intended for publication in the Pacific Journal of Mathematics should be typewritten (double spaced). The first paragraph or two must be capable of being used separately as a synopsis of the entire paper. It should not contain references to the bibliography. Manuscripts may be sent to any one of the four editors. All other communications to the editors should be addressed to the managing editor, Richard Arens at the University of California, Los Angeles, California 90024 .

50 reprints per author of each article are furnished free of charge; additional copies may be obtained at cost in multiples of 50 .

The Pacific Journal of Mathematics is published monthly. Effective with Volume 16 the price per volume (3 numbers) is $\$ 8.00$; single issues, $\$ 3.00$. Special price for current issues to individual faculty members of supporting institutions and to individual members of the American Mathematical Society: $\$ 4.00$ per volume; single issues $\$ 1.50$. Back numbers are available.

Subscriptions, orders for back numbers, and changes of address should be sent to Pacific Journal of Mathematics, 103 Highland Boulevard, Berkeley 8, California.

Printed at Kokusai Bunken Insatsusha (International Academic Printing Co., Ltd.), No. 6, 2-chome, Fujimi-cho, Chiyoda-ku, Tokyo, Japan.

PUBLISHED BY PACIFIC JOURNAL OF MATHEMATICS, A NON-PROFIT CORPORATION

The Supporting Institutions listed above contribute to the cost of publication of this Journal, but they are not owners or publishers and have no responsibility for its content or policies. 


\section{Pacific Journal of Mathematics \\ Vol. 20, No. $2 \quad$ October, 1967}

Edward Dewey Davis, Ideals of the principal class, $R$-sequences and a certain monoidal transformation ............................. 197

Richard Mansfield Dudley, Sub-stationary processes ................ 207

Newton Seymour Hawley and M. Schiffer, Riemann surfaces which are doubles of plane domains ......................... 217

Barry E. Johnson, Continuity of transformations which leave invariant certain translation invariant subspaces ................... 223

John Eldon Mack and Donald Glen Johnson, The Dedekind completion of

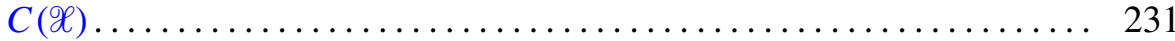

K. K. Mathur and R. B. Saxena, On the convergence of quasi-Hermite-Fejér interpolation................................... 245

H. D. Miller, Generalization of a theorem of Marcinkiewicz............ 261

Joseph Baruch Muskat, Reciprocity and Jacobi sums ............... 275

Stelios A. Negrepontis, On a theorem by Hoffman and Ramsay .......... 281

Paul Adrian Nickel, A note on principal functions and multiply-valent canonical mappings .............................. 283

Robert Charles Thompson, On a class of matrix equations ............. 289

David Morris Topping, Asymptoticity and semimodularity in projection lattices ........................................ 317

James Ramsey Webb, A Hellinger integral representation for bounded linear functionals

Joel John Westman, Locally trivial $C^{r}$ groupoids and their representations...

Hung-Hsi Wu, Holonomy groups of indefinite metrics 